\title{
HABILIDADES DE LEITURA E COMPETÊNCIAS DE COMPREENSÃO DE TEXTO DE ESTUDANTES BOLSISTAS ORIUNDOS DE COMUNIDADES DA AMAZÔNIA: ESTUDO DE CASO COM A UTILIZAÇÃO DE EYE TRACKER
}

\author{
HABILIDADES DE LECTURA Y COMPETENCIAS DE COMPRENSIÓN DE \\ TEXTO DE ESTUDIANTES CON BECAS DE LAS COMUNIDADES \\ AMAZONICAS: ESTUDIO DE CASO CON LA UTILIZACIÓN DE EYE \\ TRACKER
READING SKILLS AND TEXT COMPREHENSION COMPETENCES OF SCHOLARSHIP STUDENTS FROM INDIGENOUS AMAZONIAN COMMUNITIES: AN EYE TRACKING CASE STUDY

\author{
Gabriel LEVRINI ${ }^{1}$
}

RESUMO: O objetivo desta pesquisa é analisar as habilidades de leitura de escolares com dispositivo de rastreamento ocular de comunidades nativas da Amazônia contempladas com bolsas de estudo de universidades do Governo do Peru, utilizando técnicas biométricas, linguísticas e psicológicas. Dois grupos de escolares foram selecionados como amostra experimental: o primeiro grupo selecionou 48 estudantes bolsistas de cinco diferentes universidades públicas e privadas da Amazônia peruana; e o segundo grupo, usado como controle, continha 46 bolsistas de três universidades diferentes localizadas na região metropolitana de Lima. A pesquisa em literatura explora o potencial educativo em relação à característica individual das habilidades de compreensão de leitura, que, por definição, está relacionada ao aprendizado a partir da leitura de textos. As principais conclusões da pesquisa são: a) em média, os beneficiários de bolsas de estudos de comunidades indígenas têm uma duração de fixação maior do que os beneficiários de estudantes sediados em Lima em todas as áreas de interesse; b) em média, os beneficiados das comunidades amazônicas possuíam mais pontos de fixação em todas as áreas de interesse, o que poderia significar maior dificuldade de compreensão dos textos; em algumas das AOI, diferenças significativas foram encontradas entre os grupos; c) os bolsistas das comunidades amazônicas tiveram maior tempo de fixação e mais pontos de fixação, o que indica maior tempo de leitura e potenciais problemas de compreensão de leitura; d) Os acadêmicos também mostraram mais dificuldade em compreender textos. No entanto, provaram que seu maior esforço no processo de leitura também representa um grande sucesso maior para eles como membros da bolsa de estudos.

PALAVRAS-CHAVE: Leitura. Estudantes com bolsa de estudos da Amazônia. Eyetracking.

\footnotetext{
${ }^{1}$ Universidad del Pacifico (UP), Lima - Peru. Professor Pesquisador. Pós-doutorando na Universidade Federal do Rio Grande do Sul. ORCID: http://orcid.org/0000-0001-9992-5422. E-mail: g.levrini@up.edu.pe
} 
RESUMEN: El objetivo de esta investigación es analizar las habilidades de lectura de los académicos con dispositivos de seguimiento ocular de comunidades amazónicas nativas contempladas con becas universitarias del Gobierno del Perú utilizando técnicas biométricas, lingüísticas y psicológicas. Se seleccionaron dos grupos de escolares como muestra experimental: el primer grupo seleccionó a 48 becarios de cinco universidades públicas y privadas de la Amazonía peruana diferentes; y el segundo grupo, utilizado como control, contenía 46 becarios de tres universidades diferentes ubicadas en Lima metropolitana. La investigación literaria explora el potencial educativo en relación con las características individuales de las habilidades de comprensión de lectura, que, por definición, se relaciona con el aprendizaje de la lectura de textos. Las principales conclusiones de la investigación son: a) en promedio, los beneficiarios de becas de las comunidades indígenas tienen una mayor duración de la fijación que los beneficiarios de estudiantes con base en Lima en todas las Áreas de interés; b) en promedio, los beneficiarios de las comunidades amazónicas tenían más puntos de fijación en todas las áreas de interés, lo que podría significar una mayor dificultad para comprender los textos; en algunos AOI, se encontraron diferencias significativas entre los grupos; c) los beneficiarios de las comunidades amazónicas tuvieron una mayor duración de la fijación y más puntos de fijación, lo que indica un mayor tiempo de lectura y posibles problemas en la comprensión de la lectura; d) Los académicos también mostraron más dificultad para entender los textos. Sin embargo, demostraron que su mayor esfuerzo en el proceso de lectura también representa un mayor éxito para ellos como becarios.

PALABRAS CLAVE: Lectura. Estudiantes becarios de la Amazonia. Eye-tracking.

ABSTRACT: The objective of this research is to analyze the reading skills of scholars with eye tracking device from native Amazonian communities contemplated with universities scholarships from the Government of Peru using biometric, linguistic and psychological techniques. Two group of escolar were selected as experiment sample: the first group 48 selected scholarship students from five different Peruvian Amazon public and private universities; and the second group, used as control, contained 46 scholarship student from three different universities based in metropolitan Lima. Literature research explore educational potential in relation to the individual characteristic of reading comprehension skills, which, by definition, is related to learning from text reading.The main research conclusions are: a) on average, scholarship beneficiaries from indigenous communities have a greater fixation duration than recipients from Lima based students' in all the Areas of Interest; b) on average, grantees from Amazon communities had more fixation points in all the areas of interest, which could mean greater difficulty understanding the texts, in some AOI, significant differences were found between the groups; c) grantees from Amazon communities had greater fixation duration and more fixation points, which indicates longer reading time and potential problems in reading comprehension; d) Scholars also showed more difficulty to understanding texts. Nevertheless their proved that their major effort in the reading process also represents a major greater success for them as scholarship members.

KEYWORDS: Reading. Amazon native scholarship students. Eye-tracking. 


\section{Introdução}

Segundo o Instituto Nacional de Estatística e Informática do Peru (INEI), o censo de 2017 mostrou que a população indígena da Amazônia peruana é de 332.000 indivíduos, dos quais 52,2\% são homens e 47,8\% são mulheres. Por faixa etária, 47,5\% têm menos de 15 anos, $50,6 \%$ têm entre 15 e 64 anos e 1,9\% têm mais de 64 anos. Essas comunidades amazônicas representam 43 grupos étnicos e 13 famílias linguísticas. A preservação cultural e o desenvolvimento desses grupos étnicos é uma prioridade de inclusão social do governo peruano, especialmente em termos de saúde e educação.

O Instituto de Desenvolvimento Econômico e Empresarial (IEDEP), que administra o Índice de Desenvolvimento Humano do Peru (IDH), observou que o IDH do Peru está atualmente classificado em $84^{\circ}$ (dentre 187 países), com um país em média de 0,734 (abaixo da média do índice de 0,744). Por região, o índice do IDH da Amazônia teve uma média de 0,4846, com uma renda média anual de US\$ 435,7. Por outro lado, a capital Lima possui o maior índice de IDH do país, 0,788, com uma renda média anual de US\$1017,8 por ano. O índice IDH reflete status, gastos e desempenho em saúde, educação e renda (IEDEP, 2017).

Dispositivos especializados de neurociência, como rastreadores oculares, permitem medir as habilidades de compreensão de leitura, isto, por definição, também está relacionado ao aprendizado pela leitura de texto, em termos do registro chamado de correções (ou pontos de estabilização do olhar) que um indivíduo executa ao observar um conteúdo mostrado em uma tela. Posteriormente, séries de métricas são calculadas a partir das correções geradas para uma determinada área da tela (conhecida como Área de Interesse, AOI). As AOIs são as partes da tela através das quais passa a atenção visual dos usuários (o tempo total que eles passam olhando para uma determinada área ou o número de vezes que a consultam). Neste estudo, como pretendemos avaliar o desempenho da leitura e a compreensão do texto, criamos AOIs associadas a áreas de tela de conteúdo padronizado. Vários autores (VAN; SCHEITER, 2010; KOSKI; OLSON; NEWCOMBE, 2013) exploraram informações relevantes destinadas à transmissão através dos textos mostrados na tela do computador.

O objetivo desta pesquisa é analisar as habilidades de leitura e a compreensão de texto de universitários da Amazônia peruana em comparação com universitários de Lima por meio de técnicas biométricas, linguísticas e psicológicas e um dispositivo especializado de rastreamento ocular. Para nossa amostra, contamos com os beneficiários de bolsas de estudo concedidas pelo Programa Nacional de Bolsas de Estudo e Crédito Educacional (PRONABEC), 
com o objetivo de obter um entendimento profundo das diferenças devido às etnias dos indivíduos. Nossa hipótese foi de que as características relacionadas ao aluno e ao texto influenciam o processo de leitura, que o processo de leitura está correlacionado com a compreensão do texto e que esse é, portanto, um preditor do desempenho dos alunos. Os resultados de nossa pesquisa podem permitir ao PRONABEC tomar ações corretivas e fortalecer suas políticas de inclusão social e identificação de talentos.

\section{Revisão de literatura}

A incorporação de técnicas de rastreamento ocular pode ser um complemento ideal para o uso de técnicas clássicas de coleta de dados, como análise de eficiência por meio de materiais multimídia (HYONA, 2010). A principal vantagem de incorporar essa fonte adicional de informação reside em sua natureza objetiva. Ao ser comparada com questionários ou pesquisas, que têm um caráter mais subjetivo, a técnica de rastreamento ocular fornece informações objetivas que não são controladas conscientemente pelos sujeitos.

Já existe uma extensa literatura de pesquisa de rastreamento ocular que abrange diferentes áreas, como estudos de doenças cerebrais, avaliação de publicidade on-line e off-line e avaliação de interfaces de usuário de sites (POOLE; BALL, 2005). No campo educacional, o número de estudos é menor (MOLINA et al., 2014; MASON; TORNATORA; PLUCHINO, 2013), principalmente no caso de estudos comparativos de indivíduos de grupos minoritários.

Os movimentos oculares são estudados há mais de cinquenta anos. Somente na última década, essa medição levou a importantes descobertas sobre os processos psicológicos que ocorrem durante as tarefas de leitura, como busca visual e percepção de cena (LIVERSEDGE et al., 2006). Esses movimentos oculares servem para mover a fóvea (a parte de alta resolução da retina) que abrange 2 graus no centro do campo visual, para uma AOI, obtendo assim melhores detalhes do processo. A visão do movimento ocular (ou sacada) é geralmente suprimida, enquanto novas informações são mais comumente adquiridas durante as fixações (RAYNER, 1998).

\section{Fundamentação teórica}

Identificamos vários modelos (Figura 1) que explicam a interface entre a visão e os aspectos de baixo nível do processamento da linguagem, ou seja, modelos que especificam alguma combinação dos seguintes componentes da leitura: controle do movimento ocular, 
atenção espacial visual e processamento visual de palavras.

O modelo E-Z Reader (REICHLE et al., 1998) faz um trabalho melhor de contabilizar uma ampla gama de dados do que outros modelos. O E-Z Reader opera com base na suposição básica de que o processamento cognitivo contínuo (linguístico) influencia os movimentos dos olhos durante a leitura, basicamente, porque seu objetivo não foi desenvolvido para uma compreensão mais profunda do processo de leitura (não considera outros efeitos paralelos de processamento linguístico de nível superior nos movimentos oculares). Na opinião dos autores, o modelo E-Z Reader constitui uma simulação do que acontece quando o processo de leitura ocorre normalmente. Portanto, a implicação é que o modelo não cobre ou explica adequadamente certos problemas, como, por exemplo, regressões entre palavras.

No modelo E-Z Reader, os locais de fixação são determinados por uma combinação de fatores visuais, oculomotores e linguísticos. De acordo com o modelo, cinco processos determinam quando e para onde os olhos se movem: I) familiaridade lexical, II) conclusão do acesso lexical, III) etapas lábeis e IV) não lábeis da programação sacádica e V) dinâmica das sacadas (REICHLE et al., 1998). Na Figura 1, resumimos os principais modelos predominantes na literatura:

Figura 1 - Modelos de controle dos movimentos oculares na leitura

\begin{tabular}{|c|c|}
\hline Model & Key References \\
\hline E-Z Reader (SAS) & $\begin{array}{l}\text { Reichle, Pollatsek, Fisher, \& Rayner, } 1998 \\
\text { Reichle, Rayner, \& Pollatsek, } 2003 \text { Pollatsek, } \\
\text { Reichle, \& Rayner, } 2006\end{array}$ \\
\hline SWIFT (GAG) & $\begin{array}{l}\text { Engbert, Longtin, \& } \quad \text { Kliegl, } 2002 \\
\text { Engbert, Nuthmann, Richter, \& Kliegl, } 2005\end{array}$ \\
\hline Mr. Chips (Ideal Observer) & $\begin{array}{lllll}\text { Legge, } \quad \text { Klitz, } \quad \text { \& } \quad \text { Tjan, } & 1997\end{array}$ \\
\hline & $\begin{array}{l}\text { Legge, Hooven, Klitz, Mansfield, \& Tjan, } 2002 \\
\text { Salvucci, } 2001\end{array}$ \\
\hline EMMA (SAS within ACT system) & Reilly \& Radach, 2006 \\
\hline $\begin{array}{l}\text { Glenmore (GAG within Connectionist System) } \\
\text { SERIF (POC) }\end{array}$ & McDonald, Carpenter, \& Shillcock, 2005 \\
\hline Competition/Activation (POC) & \\
\hline & $\begin{array}{l}\text { Yang } \\
\text { Yang, 2006 }\end{array}$ \\
\hline & Feng, 2006 \\
\hline SHARE (POC) & \\
\hline
\end{tabular}

Fonte: Elaborado pelo autor adaptado de Rayner (2009). 
Na Tabela 1, é possível comparar as características dos movimentos oculares durante a leitura, a percepção da cena e a busca visual. Pode-se inferir que os valores apresentados são bastante representativos das diferentes tarefas, embora exista considerável variabilidade no intervalo médio das durações de fixação (durações de fixação e comprimentos das sacadas). Durante o processo de leitura, a duração da fixação é de cerca de $225-250 \mathrm{~ms}$, e o tamanho da sacada geralmente ocupa 8 a 9 espaços de caracteres (RAYNER, 1998).

Tabela 1 - Características dos movimentos oculares durante a leitura, percepção da cena e busca visual

\begin{tabular}{clc}
\hline \multicolumn{1}{c}{ Tarefa } & $\begin{array}{l}\text { Duração } \\
\text { média típica } \\
\text { da fixação }(\mathrm{ms})\end{array}$ & $\begin{array}{l}\text { Tamanho médio da sacada } \\
\text { (graus) }\end{array}$ \\
\hline Leitura silenciosa & $225-250$ & 2 (8-9 espaços de caractere) \\
Leitura oral & $275-325$ & $1.5(6-7$ espaços de caractere) \\
Percepção da cena & $260-330$ & 4 \\
Busca visual & $180-275$ & 3 \\
\hline
\end{tabular}

Fonte: Rayner (1998).

\section{Movimentos oculares: lendo e pulando a leitura}

Durante a leitura, os olhos seguem certos padrões específicos conhecidos como sacadas e fixações. Os movimentos oculares são considerados um reflexo do processo cognitivo (RAYNER, 1998). Em geral, o interesse do leitor é revelado por uma fixação. A recuperação de informações na leitura é feita durante as fixações; nenhuma informação é obtida durante uma sacada (entre duas fixações) (RAYNER, 2009).

Na leitura, diferentemente de outras tarefas visuais, os espaços de caracteres são usados em vez de ângulos visuais. Isso ocorre porque foi demonstrado (CASTELHANO; HENDERSON, 2007) que os espaços de caracteres são uma unidade de medida mais apropriada que o ângulo visual. O movimento característico dos leitores (que no caso da leitura ocorre cerca de 10 a 15\% do tempo), é que os leitores movem os olhos (regressão) de volta às palavras anteriores no texto. A frequência das regressões geralmente depende da dificuldade do texto (CASTELHANO; HENDERSON, 2007; MORRISON; RAYNER, 1981), vocabulário e memória de trabalho. Além disso, o tamanho da sacada e a duração da fixação são modulados pela dificuldade do texto: à medida que o texto se torna mais difícil, o tamanho da sacada tende a diminuir, a duração da fixação tende a aumentar e as regressões tendem a aumentar. A partir 
dessas medidas, fica claro que as propriedades gerais do texto influenciam significativamente os movimentos dos olhos. Em outras palavras, o tipo de material que está sendo lido e o objetivo básico do leitor em ler (RAYNER, 2009) também influenciam medidas globais como tamanho da sacada, duração da fixação e o número de regressões (ESKENAZI; FOLK, 2015; WILLIAMS; MORRIS 2004).

Uma questão importante a considerar ao medir a leitura é a quantidade de informações que o leitor é capaz de processar e usar durante uma única fixação (em torno de 200-250 ms). Essa medida é denominada campo de visão funcional (WILLIAMS; MORRIS, 2004), também influenciada por vários fatores linguísticos (Figura 2):

Figura 2 - Fatores linguísticos como Influenciadores de tempo ${ }^{2}$

\begin{tabular}{|ll|}
\hline Factor & \multicolumn{1}{c|}{ Authors } \\
Frequency of the fixated word & Inhoff \& Rayner, 1986; Rayner \& Duffy, 1986 Ehrlich \& \\
Predictability of the fixated word & Rayner, 1981; Rayner \& Well, 1996 \\
Meanings of the fixated word & Sereno, O'Donnell, \& Rayner, 2006 \\
Acquisition of the word meaning & Juhasz \& Rayner, 2006 \\
Semantic relations between words & Carroll \& Slowiaczek, 1986; Morris, 1994 \\
Familiarity of the word & Williams \& Morris, 2004 \\
& \\
& \\
\end{tabular}

Fonte: Elaborado pelos autores com base em Castellano e Henderson (2007).

Os leitores adultos pulam aproximadamente um terço das palavras em um texto enquanto leem (RAYNER, 1998; 2009). Existem duas visões diferentes sobre o que leva à decisão de quando mover os olhos. A visão dominante é que depende inteiramente do processamento de texto em andamento: a decisão de mover os olhos é tomada apenas quando o processamento da palavra atualmente fixada atinge um certo ponto (REICHLE; RAYNER; POLLATSEK, 2003) e quando uma proporção das sacadas são acionadas autonomamente após um certo período de processamento de texto em andamento (ESKENAZI; FOLK, 2015).

Os olhos dos leitores frequentemente pulam as palavras enquanto leem. As taxas de pulo são amplamente determinadas pelo tamanho da palavra; palavras curtas são mais puladas que

${ }^{2}$ Se lê na imagem: Primeira linha - Fator / Autores; segunda linha - frequência da palavra fixada; terceira linha Previsibilidade da palavra fixada; quarta linha - Significados da palavra fixada; quinta linha - Aquisição do significado da palavra; sexta linha - Relações semânticas entre as palavras; sétima linha - Familiaridade com a palavra.

RIAEE - Revista Ibero-Americana de Estudos em Educação, Araraquara, v. 15, n. 3, p. 1094-1114, jul./set. 2020. e-ISSN: 1982-5587. 
palavras longas. No entanto, a previsibilidade de uma palavra no contexto também afeta as taxas de pulos. (REICHLE; DRIEGUE, 2015). Para ler com eficiência, os olhos devem permanecer fixos nas palavras, apenas o tempo suficiente para a identificação. No entanto, as palavras diferem em termos de quão fáceis são de processar e, portanto, quanto tempo precisam ser fixadas durante a leitura (RAYNER, 1998; 2009). Para finalizar uma fixação, é necessário programar uma sacada para um novo local, que leva aproximadamente $125-150 \mathrm{~ms}$. Portanto, um processo de leitura eficiente exige que os leitores aprendam a programar os movimentos dos olhos para novos locais antes da conclusão do processamento de texto em andamento. Caso contrário, os olhos permanecerão em locais por mais tempo do que o ideal (RAYNER, 2009).

A questão do salto de palavras diz respeito à decisão relacionada aos movimentos oculares de primeira passagem, avanço e passagem entre palavras. Ambos os modelos, o E-Z Reader (REICHLE; DRIEGUE, 2015) e SWIFT (ENGBERT et al., 2005), preveem que o salto de palavras pode ocorrer com base no processamento lexical incompleto da palavra seguinte, embora por razões diferentes. No caso do EZ Reader, os pulos pretendidos de uma palavra futura $(n+1)$ serão acionados quando o estágio inicial do processamento lexical (L1) da palavra $\mathrm{n}+1$ for concluído antes do estágio inicial do processamento oculomotor (M1) da palavra $\mathrm{n}$ ser concluído. No entanto, com SWIFT, os pulos pretendidos ocorrem quando a ativação lexical da palavra $\mathrm{n}+2$ (ou além) é maior que a ativação lexical da palavra $\mathrm{n}+1$. Portanto, com o E-Z Reader, o fator importante é a quantidade de processamento lexical da próxima palavra que ocorreu, mas para o modelo SWIFT, o fator importante é o grau relativo de processamento lexical das próximas palavras. A palavra selecionada depende do comprimento das palavras e sua distância da posição atual de fixação (POLLATSEK; REICHLE; RAYNER, 2006; LITZINGER et al., 2007).

\section{Compreensão de texto e modelos mentais}

Os movimentos oculares fornecem evidências sobre a relação entre as características cognitivas e linguísticas dos alunos, como decodificação de palavras, vocabulário, habilidade de compreensão, memória de curto prazo, memória de trabalho, inteligência não-verbal (LEEW; SEGERS; VERHOEVEN, 2015) e compreensão de texto (VAN DEN BROEK et al., 2005; JUST; CARPENTER, 1980). Os resultados mostram que os efeitos diferenciais das características do leitor e do texto afetam a probabilidade de pular, impulsionada pela decodificação e pela inteligência não-verbal. Um pré-requisito para a compreensão de texto é a construção de um modelo mental coerente (KINTSCH, 2004) com decodificação influenciada 
pela probabilidade de regressão e duração do caminho de regressão, mostrando que o comportamento de leitura está relacionado às habilidades do aluno e às medidas de compreensão de texto. Em suma, o comportamento de leitura tende a estar relacionado às habilidades dos alunos e às medidas de compreensão de texto (BLANC et al., 2008).

\section{Modelos mentais}

O descanso e a atualização dos modelos mentais ocorrem, criando vínculos entre as proposições com o auxílio de inferências geradas por meio de informações dentro do modelo mental atual (inferências baseadas na memória) ou conhecimento prévio (inferências elaboradas) (VAN DEN BROEK; RAPP; KENDEOU, 2005). Modelos mentais coerentes são construídos durante a leitura, por meio da atualização constante do modelo atual (KINTSCH, 2004). Além disso, as habilidades e capacidades dos leitores também influenciam a construção de modelos mentais. Um maior conhecimento de vocabulário ajuda o leitor a entender melhor os conceitos contidos no texto, o que, por sua vez, aumenta a chance de inferências baseadas na memória (CALVO et al., 2001), portanto, como o vocabulário está relacionado ao conhecimento do mundo, um vocabulário mais amplo também aumenta a chance de fazer inferências elaboradas e vincular o texto ao conhecimento prévio (VAN DEN BROEK et al., 2005).

A compreensão de texto está altamente relacionada ao número de inferências geradas durante a leitura, portanto memória de curto prazo e de trabalho são preditores importantes da compreensão de leitura (CAIN et al., 2001). Pesquisas anteriores descobriram que a inteligência não verbal e os níveis de compreensão estão correlacionados com o desenvolvimento dos leitores para prever a compreensão de leitura para além das habilidades de linguagem e memória (TIU; THOMPSON; LEWIS, 2003)

\section{Características dos leitores}

A literatura mostra que as características dos alunos afetam os processos de compreensão de leitura (RAYNER, 1985). Os efeitos relacionados ao texto podem variar em função de várias características relacionadas ao aluno, como efeitos de comprimento e frequência de palavras. Além disso, os leitores não prestam igual atenção a todas as palavras (KINTSCH, 2004) e os leitores com desenvolvimento hábil dedicam mais tempo a elementos importantes do texto, como cabeçalhos, resumos, etc. (VAN; SCHEITER, 2010). Portanto, a 
maneira como os leitores alocam sua atenção depende de ambos, da palavra e de sua posição na sentença e no parágrafo (CALVO et al., 2001).

$\mathrm{O}$ potencial educacional associado às características de habilidades de leitura e compreensão dos indivíduos está relacionado ao aprendizado através do texto (CROMLEY; SNYDER-HOGAN; LUCIW-DUBAS; 2010; MASON et al., 2015; LEEUW; SEGERS; VERHOEVEN, 2015). Com base nisso, formulamos as seguintes hipóteses:

\section{H1 - A característica relacionada ao aluno influencia o processo de leitura, dentro dos dois grupos}

Como observamos, o IDH médio da Amazônia é de 0,4846, enquanto a renda média anual é de US\$ 435,7, abaixo da média nacional nos dois casos. Historicamente, os governos negligenciaram a região e somente nas últimas décadas a inclusão social foi priorizada e programas foram desenvolvidos com o objetivo de mudar esse padrão. Entretanto, estratégias educacionais governamentais, especialmente em áreas subdesenvolvidas como a Amazônia, são menos eficazes devido a variáveis sociodemográficas.

\section{H2 - Estudantes indígenas da Amazônia terão mais tempo de fixação em comparação com estudantes de Lima}

O contexto faz com que as fixações sejam interpretadas de maneiras diferentes. Por exemplo, ao navegar em uma página da web, uma taxa mais alta de fixações em uma área específica pode mostrar um interesse maior nessa área fixada. Também pode ser uma indicação de que a área é de alguma forma mais complexa. Essas interpretações podem ser diferentes se as fixações ocorrerem durante uma tarefa de pesquisa, por exemplo; nesse caso, uma maior taxa de fixação pode indicar maior incerteza ao tentar reconhecer um item (POOLE; BALL, 2005).

\section{H3 - Estudantes indígenas da Amazônia terão pontos de fixação mais longos, refletindo dificuldades na compreensão ou falta de significado do texto para o leitor}

A dificuldade de uma palavra é expressa na duração das fixações. Quando um leitor encontra uma palavra desconhecida, ele ou ela fixa mais a palavra. Por outro lado, se a palavra tiver uma frequência alta ou familiar, a fixação na palavra será mais curta. No entanto, o leitor pode encontrar uma palavra ou sintaxe problemática para ele ou ela. Quando isso acontece, o número de fixações em torno dessa palavra e a duração das fixações aumentam, enquanto o leitor tenta compreendê-la (HYRSKYKARI et al., 2000). Prevemos que a eficiência da decodificação de palavras terá fortes efeitos nos movimentos oculares para estudantes indígenas 
da Amazônia, pois a decodificação de palavras está altamente relacionada à velocidade da leitura (e à duração dos movimentos oculares). Com relação aos efeitos da posição das palavras, esperávamos que os leitores com mais habilidades passassem mais tempo na integração de texto e nas regiões de texto mais salientes (ou seja, cabeçalho e primeira frase de um parágrafo). Além disso, esperamos que a memória de trabalho preveja o comportamento da regressão e os resultados da compreensão de leitura porque um curto período de memória limita a quantidade de informações disponíveis para manter a coerência (por exemplo, gerando inferências).

\section{Material e métodos}

\section{Aparelho}

Para registrar os movimentos oculares dos alunos, usamos um dispositivo Tobii X2-30, fabricado pela Tobii Technology e usando o software Tobii pro3.4.8 (TOBII, 2014). Este dispositivo funciona com uma frequência de amostragem de $30 \mathrm{~Hz}$ e tem uma resolução espacial inferior a $0,5^{\circ}$. Ligamos o rastreador ocular a um monitor TFT de 24 polegadas com uma resolução máxima de 1920 x 1200 pixels. O sistema permite amplos movimentos da cabeça, proporcionando um ambiente livre de distrações para garantir o comportamento natural e, portanto, resultados válidos. A tecnologia de rastreamento de alta precisão garante que os resultados da pesquisa sejam confiáveis (TOBII, 2014). As margens do texto eram de 200 pixels (px) de todos os lados da tela. A fonte era Arial, 20 px e altura da linha 3 em estilo romano. Os títulos foram apresentados em fonte semelhante, mas os títulos foram impressos em negrito, com 30 px, altura da linha 2 e subtítulos em 20 px, altura da linha 2. Vários estudos (KOSKI; OLSON; NEWCOMBE, 2013; LAGUN et al., 2014; MASON et al., 2013; MOLINA et al., 2014) usaram o mesmo dispositivo e software similar com uma presença maior ou menor de elementos multimídia.

\section{Características relacionadas ao aluno}

Eficiência de decodificação: Medimos a eficiência da decodificação usando uma tarefa de leitura de palavras (JONGEN; KROM, 2009). Nesta tarefa, apresentamos aos alunos um cartão de 120 palavras, dividido em quatro colunas, e os instruímos a ler em voz alta o maior número possível de palavras em um minuto. Demos aos alunos um ponto por cada palavra lida corretamente. A consistência interna do teste foi classificada como boa $(\alpha=.85)$ (EGBERINK; JANSSEN; VERMEULEN, 2014). 
Conhecimento de vocabulário: Testamos o conhecimento do vocabulário usando o teste de conhecimento do vocabulário passivo padronizado (Vocabulário para Leitura adaptado de VERHOEVEN; VERMEER, 1999). Este teste consiste em 30 itens de múltipla escolha, nos quais cada palavra foi apresentada em um contexto curto. Pedimos aos alunos o significado da palavra sublinhada nas diferentes opções apresentadas, que incluíam um sinônimo da palavra alvo.

Memória: Utilizamos uma tarefa de memória baseada na Escala de Inteligência Wechsler (KORT, et al., 2005), na qual lemos em voz alta uma sequência de dígitos usando uma entonação decrescente e fazendo uma pausa por um segundo entre os dígitos. Em resposta, os alunos foram instruídos a lembrar os dígitos na mesma ordem em que foram apresentados. No final, administramos uma tarefa de repetição de sentenças para medir a memória de informações sintáticas (VERHOEVEN, et al., 2013).

Habilidades de compreensão: Medimos as habilidades de compreensão usando um teste padronizado (FEENSTRA, 2008), composto por cinco textos e 25 perguntas de múltipla escolha. Os textos eram narrativos e expositivos e incluíam uma mistura de perguntas baseadas em texto e em inferência.

Inteligência não verbal: Para avaliar a inteligência não verbal, foram utilizadas as Matrizes Progressivas Padrão (SPM, abreviação em inglês) (RAVEN, 1981). Este é um teste de múltipla escolha, com 60 itens que aumentam em dificuldade, com exercícios como identificar o elemento ausente para completar um padrão mostrado em uma figura específica.

\section{Design da experiência}

Pré-teste: Individualmente, 120 estudantes de faculdades da região metropolitana de Lima e da Amazônia fizeram um pré-teste que consistia em uma série de perguntas abertas, cujo objetivo era determinar o conhecimento prévio do conteúdo a ser distribuído posteriormente no processo. Como era de se esperar, dada a natureza do conteúdo sociológico, nenhum aluno demonstrou ter conhecimento sobre o conteúdo.

Aplicamos o teste de QI de Raven e, embora a média (127,5) tenha sido 5,1 pontos mais baixa para os estudantes da Amazônia, quando comparada com os estudantes de Lima (DP = 14 e variação de 100-140), o total de pontos de teste foi de acordo com as expectativas para o uso da amostra. Em seguida, selecionamos dois grupos contendo indivíduos de ambos os sexos, com idades entre 17 e 20 anos. O primeiro grupo era formado por 48 peruanos da Amazônia indígena de cinco faculdades públicas; enquanto o segundo grupo tinha 46 acadêmicos de três 
faculdades públicas diferentes na região metropolitana de Lima. O experimento ocorreu de junho a novembro de 2018 nas faculdades selecionadas.

Em seguida, pedimos ao aluno que se sentasse em frente ao monitor para realizar a calibração do rastreador ocular. A tela exibiu, por 15 minutos, um texto sociológico de 250 palavras, intitulado "Pensamento filosófico e felicidade desde seu princípio na Grécia", enquanto o rastreador registrava as fixações feitas pelos alunos durante a atividade.

Pedimos aos alunos que respondessem a duas perguntas sobre o conteúdo do texto, cada uma com dez respostas possíveis de múltipla escolha. Finalmente, uma vez que eles concluíram o teste, os alunos tiveram que preencher outro questionário destinado a medir até que ponto eles haviam assimilado o conteúdo. O tempo total necessário para cada aluno concluir todas as fases foi de aproximadamente 25 minutos.

Utilizamos o teste t de Student para comparar as duas amostras de estudantes. Usamos um teste de duas amostras de Kolmogorov-Smirnov para avaliar a distribuição normal dos dois grupos. Embora as pontuações nos dois papéis sejam ligeiramente diferentes, não encontramos nenhuma diferença significativa entre os dois grupos (grupo de rastreamento ocular da Amazônia indígena vs. grupo de rastreamento ocular baseado em Lima) $(\mathrm{D}=0.112, \mathrm{p}=.97)$.

Realizamos a mesma análise para testar possíveis diferenças nos tempos de resposta, mas, novamente, não encontramos diferença entre os dois grupos $(\mathrm{D}=0.147, \mathrm{p}=.36)$.

\section{Resultados}

Tabela 2 - Características do aluno

\begin{tabular}{lcccccc}
\hline & & \multicolumn{5}{c}{ Valores } \\
Características do aluno & Indig.Amaz & Lima & $\boldsymbol{M}$ & $\boldsymbol{P}$ & $\boldsymbol{D P}$ & Alcance \\
\hline 1. Habilidades de decodificação & 0.68 & 0.71 & 73.9 & 0.221 & 12.6 & 53 a 116 \\
2. Vocabulário & -0.03 & $.356^{* *}$ & 20 & $0.013^{* *}$ & 3.6 & 10 a 27 \\
3. Memória de curto prazo & 0.002 & 0.0031 & 12.5 & 0.223 & 2 & 7 a 16.1 \\
4. Memória de trabalho & -0.067 & -0.065 & 4.1 & -0.143 & 1.2 & 2 a 7 \\
5. Compreensão de leitura & 0.095 & $.177^{* *}$ & 32.3 & $.0031^{*}$ & 12.7 & 6 a 60 \\
6. Inteligência não verbal & -0.253 & $.145^{* *}$ & 42.4 & $.0042^{*}$ & 5.6 & 31 a 52 \\
7. Compreensão de texto & -0.242 & $.210^{* *}$ & 18.7 & .0102 & 3.2 & 8 a 23 \\
\hline *p $>0.010 * *$ p $>0.005$ & & & & & &
\end{tabular}

Fonte: Elaborado pelos autores (2019).

Os resultados do teste de características do aluno são mostrados na Tabela 2 acima. Como esperávamos, foram encontradas diferenças significativas entre os grupos em vocabulário, compreensão de leitura e inteligência não verbal, mas, para nossa surpresa, os 
resultados para diferenças médias na compreensão de texto entre os grupos não foram significativos. Os resultados provavelmente estão relacionados aos esforços individuais feitos pelos estudantes da Amazônia para obter boas notas, considerando que a elegibilidade para as bolsas do PRONABEC exige boas notas.

\section{Áreas de interesse (AOI)}

Para a AOI, selecionamos a sentença de texto como a unidade de análise e dividimos em nove áreas de interesse (Figura 3). Foram excluídos os participantes para quem o rastreador ocular estava mal calibrado (três do grupo alvo e nove do grupo controle).

Figura 3 - Texto da experiência e AOIs definidas

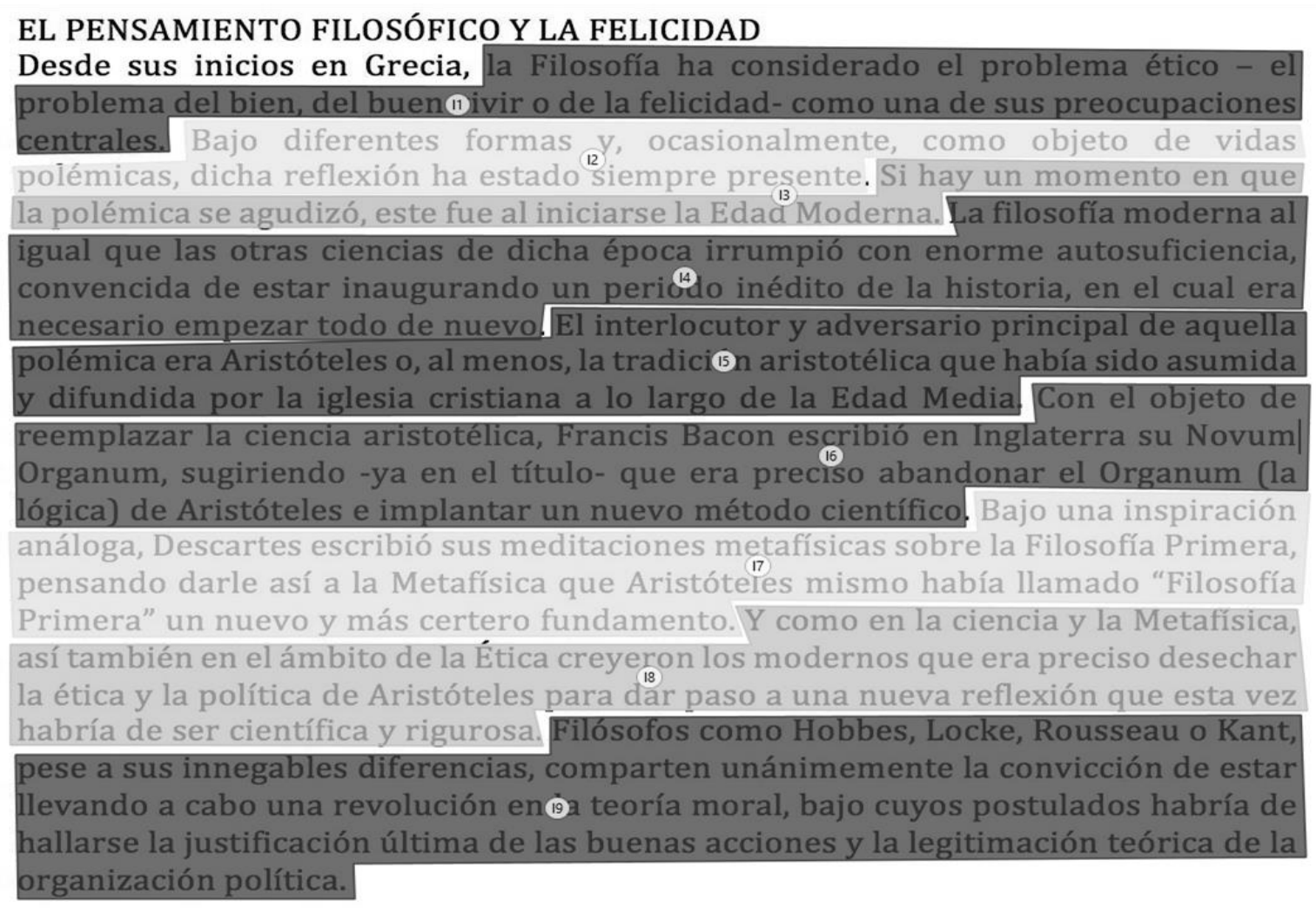

Fonte: Texto de Ética Aristotélica adaptado pelos Autores (2018). 
O texto apresentava as características estabelecidas na Tabela 3 abaixo:

Tabela 3 - Características do texto

\begin{tabular}{l|c}
\hline AOI & 9 \\
\hline $\begin{array}{l}M \text { de comprimento da palavra em } \\
\text { caracteres }\end{array}$ & 9.22 \\
\hline $\mathrm{N}^{\circ}$ de frases - AOIs & 9 \\
\hline $\begin{array}{l}M \text { de comprimentos das frases em } \\
\text { palavras }\end{array}$ & 36.71 \\
\hline Palavra nos títulos & 10 \\
\hline
\end{tabular}

Fonte: Elaborado pelos autores (2019).

Uma visão geral dos resultados das fixações e do tempo médio de fixação é fornecida na Tabela 4 e Tabela 5:

Tabela 4 - Duração da fixação

\begin{tabular}{|c|c|c|c|}
\hline AOI & Média(s) & Valor P & Sig. \\
\hline AOI 1 & $\begin{array}{l}\text { Indig. Amaz }=0.1955528 \\
\text { Controle }=0.1927273\end{array}$ & 0.6189 & \\
\hline AOI 2 & $\begin{array}{l}\text { Indig. Amaz }=0.2103571 \\
\text { Controle }=0.1937931\end{array}$ & 0.0754 & $*$ \\
\hline AOI 3 & $\begin{array}{l}\text { Indig. Amaz }=0.2048276 \\
\text { Controle }=0.1992857\end{array}$ & 0.322 & \\
\hline AOI 4 & $\begin{array}{l}\text { Indig. Amaz }=0.1961290 \\
\text { Controle }=0.1965517\end{array}$ & 0.2841 & \\
\hline AOI 5 & $\begin{array}{l}\text { Indig. Amaz }=0.1961523 \\
\text { Controle }=0.1810332\end{array}$ & 0.05 & $*$ \\
\hline AOI 6 & $\begin{array}{l}\text { Indig. Amaz }=0.1796296 \\
\text { Controle }=0.1793103\end{array}$ & 0.9816 & \\
\hline AOI 7 & $\begin{array}{l}\text { Indig. } \text { Amaz }=0.171220 \\
\text { Controle }=0.1677484\end{array}$ & 0.8129 & \\
\hline AOI 8 & $\begin{array}{l}\text { Indig. Amaz }=0.194285 \\
\text { Controle }=0.1736842\end{array}$ & 0.3159 & \\
\hline AOI 9 & $\begin{array}{l}\text { Indig. Amaz }=0.25428 \\
\text { Controle }=0.16540\end{array}$ & 0.04199 & $*$ \\
\hline
\end{tabular}

$* \mathrm{p}>0.010 * * \mathrm{p}>0.005$

Fonte: Elaborado pelos autores (2019).

Tabela 5 - Tempo médio por ponto de fixação

\begin{tabular}{lcll}
\hline AOI & $\begin{array}{c}\text { Média de tempo } \\
\text { (ponto de fixação) }\end{array}$ & Valor P & Sig. \\
\hline AOI 1 & $\begin{array}{c}\text { Indig.Amaz }=37.63703 \\
\text { Controle }=37.95238\end{array}$ & 0.999 \\
\hline AOI 2 & $\begin{array}{c}\text { Indig.Amaz }=39.21429 \\
\text { Controle }=35.68966\end{array}$ & 0.5622 \\
\hline AOI 3 & $\begin{array}{c}\text { Indig.Amaz }=24.03448 \\
\text { Controle }=22.13010\end{array}$ & 0.4276 \\
\hline AOI 4 & $\begin{array}{c}\text { Indig.Amaz }=72.13793 \\
\text { Control }=61.16129\end{array}$ \\
\hline
\end{tabular}

Indig.Amaz. $=52.9861 \quad 0.9756$

RIAEE - Revista Ibero-Americana de Estudos em Educação, Araraquara, v. 15, n. 3, p. 1094-1114, jul./set. 2020. e-ISSN: 1982-5587. 


\begin{tabular}{lcc} 
AOI 5 & Controle $=52.84311$ & \\
\hline AOI 6 & $\begin{array}{c}\text { Indig.Amaz }=51.33333 \\
\text { Controle }=57.72414\end{array}$ & 0.2478 \\
\hline AOI 7 & $\begin{array}{c}\text { Indig.Amaz }=44.0901 \\
\text { Controle }=43.88567\end{array}$ & 0.9584 \\
\hline AOI 8 & $\begin{array}{c}\text { Indig.Amaz }=53.21053 \\
\text { Controle }=49.66667\end{array}$ & 0.5113 \\
\hline AOI 9 & $\begin{array}{c}\text { Indig.Amaz }=55.11507 \\
\text { Controle }=53.90476\end{array}$ \\
\hline
\end{tabular}

$* \mathrm{p}>0.010 * * \mathrm{p}>0.005$

Fonte: Elaborado pelos autores (2019).

\section{Compreensão de texto}

No final da sessão, todos os alunos responderam a duas perguntas sobre o conteúdo do texto, cada uma com dez possíveis respostas de múltipla escolha. Os resultados são os seguintes (Tabela 6):

Tabela 6 - Respostas corretas dos alunos

\begin{tabular}{l|c}
\hline Questão 1 & $M$ \\
\hline Estudantes Indig. Amazônia & 14 \\
\hline Estudantes de Lima & 13 \\
\hline Questão 2 & \\
\hline Estudantes Indig. Amazônia & 15 \\
\hline Estudantes de Lima & 16 \\
\hline
\end{tabular}

Fonte: Elaborado pelos autores (2019).

Foi realizado um teste de correlação biserial para mostrar as correlações entre os pontos de fixação e as duas perguntas respondidas pelos alunos após a aplicação do teste de tela. Este teste é amplamente utilizado em experimentos para determinar a correlação entre duas variáveis; no nosso caso, aquela medida usando a escala de intervalo (AOI) e uma variável dicotômica (tempo) (Figura 4). 
Figura 4 - Correlações bisseriais ${ }^{3}$

\author{
Amazon Native Scholarship students \\ Fixations points AOI x Answer question No 1, r $=-0.01268$ \\ $\mathrm{p}>0.322$ \\ Fixations points AOI x Answer question No 2, $r=0.4412$ \\ $p>0.145$ \\ Lima based Scholarship students \\ Fixations points AOI x Answer question No 1, $r=0.01992$ \\ $\mathrm{p}>0.233$ \\ Fixations points AOI x Answer question No 2, $r=-0.4488$ \\ $\mathrm{p}>0.189$
}

Fonte: Elaborado pelos autores (2019).

Os resultados da correlação bisserial mostram uma correlação próxima, pois os valores estão próximos de 0 , embora não sejam significativos, pois o valor de p é superior a 0,10 .

\title{
Conclusões
}

Nosso objetivo neste estudo foi analisar as habilidades de leitura de estudantes de comunidades indígenas da Amazônia, que receberam bolsas do PRONABEC usando técnicas biométricas, linguísticas e psicológicas, a fim de possibilitar ações corretivas e fortalecer as políticas de inclusão educacional e identificação de talentos.

É bastante claro na literatura que o tamanho da sacada e a duração da fixação são modulados pela dificuldade do texto, pela quantidade de informações que o leitor é capaz de processar e pelo seu uso durante uma única fixação. Além disso, também está bem estabelecido que a dificuldade de uma palavra é expressa pelo tempo de duração das fixações, quando um leitor encontra uma palavra desconhecida. Nossa pesquisa é apoiada pela literatura sobre potencial acadêmico, em relação às características individuais relativas às habilidades de

${ }^{3}$ Lemos na figura: Primeira linha - Estudantes bolsistas indígenas na Amazônia; segunda linha - Pontos de fixação AOI x Resposta da questão n 1 ; terceira linha - Pontos de fixação AOI x Resposta da questão no 2; quarta linha Estudantes bolsistas em Lima; quinta linha - Pontos de fixação AOI x Resposta da questão nº 1 ; sexta linha Pontos de fixação AOI x Resposta da questão $n^{\circ} 2$.

RIAEE - Revista Ibero-Americana de Estudos em Educação, Araraquara, v. 15, n. 3, p. 1094-1114, jul./set. 2020. e-ISSN: $1982-5587$. 
compreensão de leitura que, por definição, estão relacionadas à aprendizagem por meio da leitura de texto. De nossos resultados, descobrimos que:

- H1 é parcialmente comprovado, pois as diferenças entre os grupos foram encontradas apenas no vocabulário, compreensão de leitura e inteligência não verbal, mas, para nossa surpresa, os resultados para as diferenças médias na compreensão de texto entre os grupos não foram significativos,

- O H2 é comprovado apenas parcialmente, pois apenas o AOI 4 foi significativo (com $\mathrm{p}$ $<.10)$.

- O H3 é novamente parcialmente comprovado, pois apenas o AOI 2, 5 e 9 foram estatisticamente significativos (com $\mathrm{p}<.10)$. Segundo Hyrskykari et al. (2000), o número de fixações em torno de palavras e a duração das fixações aumentam quando o leitor tenta entender o texto.

- A partir desta pesquisa, as principais conclusões podem ser resumidas da seguinte forma:

- Em média, os bolsistas das comunidades amazônicas têm uma duração de fixação mais longa e mais pontos de fixação, em toda a AOI, do que os estudantes de Lima, o que pode significar maior tempo de leitura e possíveis problemas na compreensão da leitura e maior dificuldade em entender o texto.

- Os bolsistas das comunidades amazônicas têm uma correlação positiva moderada, o que significa que quanto maior o número de fixações, melhor a resposta. No entanto, uma explicação para os resultados poderia ser que seu maior esforço no processo de leitura também representa maior sucesso para eles como bolsistas.

- Confirmamos com este estudo a contribuição de técnicas psicológicas para o estudo de diferentes respostas individuais na leitura.

\section{Contribuição e implicações práticas}

Essa avaliação empírica envolveu uma comparação de estudantes de diferentes origens étnicas através da medição de suas características e capacidades de aprendizagem. Os resultados são de grande importância verificada empiricamente e podem ser úteis na adaptação das políticas públicas de educação do Peru, com o objetivo de alcançar resultados de qualidade para com os objetivos educacionais. Além disso, os resultados são úteis em termos de utilização, eficiência e aplicação de recursos públicos em benefício da sociedade. Finalmente, a contribuição e os benefícios acadêmicos incluem os seguintes: 
- A tecnologia de rastreamento ocular tem o potencial de ser usada como uma tecnologia educacional. Este estudo sugere que ela pode ser usada para modelar algumas das atividades de aprendizagem mais comuns no contexto escolar.

- O benefício potencial de um exemplo de modelagem de movimento ocular parece se estender a um aprendizado mais profundo a partir da compreensão de texto e dos recursos de aprendizado.

\section{REFERÊNCIAS}

BLANC, N.; KENDEOU, P.; VAN DEN BROEK, P. W.; BROUILLET, D. Updating situation models during reading of news reports: evidence from empirical data and simulations. Discourse Processes, v. 45, n. 2, p. 103-121, 2008. DOI: 10.1080/01638530701792784

CAIN, K.; OAKHILL, J.; BARNES, M. A.; BRYANT, P. E. Comprehension skill, inferencemaking ability, and their relation to knowledge. Memory \& Cognition, v. 29, n. 6, p. 850-859, 2001. DOI: 10.3758/BF03196414

CALVO, M. G.; MESENGUER, E.; CARREIRAS, M. Inferences about predictable events: eye movements during reading. The Spanish Journal of Psychology, v. 5, n. 1, p. 66-77, jun. 2001. DOI: $10.1017 /$ S1138741600005849

CASTELHANO, M. S.; HENDERSON, J. M. Initial scene representations facilitate eye movement guidance in visual search. Journal of Experimental Psychology: Human Perception and Performance, v. 33, n. 4, p. 753-763, 2007. DOI: 10.1037/00961523.33.4.753

CROMLEY, J.G.; SNYDER-HOGAN, L. E.; LUCIW-DUBAS, U. A. Reading comprehension of scientific text: a domain-specific test of the direct and inferential mediation model of reading comprehension. Journal of Educational Psychology, v. 102, n. 3, 687-700, 2010. DOI: 10.1037/a0019452.

ESKENAZI, M. A.; FOLK, J. R. Reading skill and word skipping: Implications for visual and linguistic accounts of word skipping. Journal of Experimental Psychology: Learning, Memory, and Cognition, v. 41, n. 6, p. 1923-1928, 2015. DOI: 10.1037/xlm0000156

ENGBERT, R.; NUTHMANN, A; RICHTER E.M.; KLIEGL, R. Swift: a dynamical model of saccade generation during reading. Psychol. Review, v. 112, n. 4, p. 777-813, out. 2005. DOI: 10.1037/0033-295X.112.4.777

FEENSTRA, H. Begrijpend lezen groep 6 [Reading Comprehension Grade 4]. Arnhem, the Netherlands: Cito, 2008.

HYRSKYKARI, A.; MARAJANTA, P.; AALTONEN, A.; KARI-JOUKO RAIHA Design issues of idict: a gaze-assisted translation aid. Proceedings of the 2000 Symposium on Eye Tracking Research \& Applications, p. 9-14, 2000. 
HYONA, J. The use of eye movements in the study of multimedia learning. Learning and Instruction, v. 20, n. 2, p. 172-176, 2010. DOI: 10.1016/j.learninstruc.2009.02.013

IEDEP. Instituto de Economia y Desarollo Empresarial. Relatoria del Indice IDH para las provincias peruanas. Lima, Perú, 2017.

INEI. Instituto Nacional de Estadística e Informatica. Census Peru 2016. Lima, Perú, 2017.

JONGEN, I.; KROM, R. DMT en AVI: AVI Handleiding [DMT and AVI: AVI manual] Arnhem, the Netherlands: Cito, 2009.

JUST, M. A.; CARPENTER, P. A. The psychology of reading and language comprehension. Newtown, MA: Allyn and Bacon, 1987.

KORT W.; SCHITTEKATTE, M.; DEKKER, P.H.; VERHAEGHE, P.; Compaan EL, Bosmans M, Vermeir G. Wechsler intelligence scale for children derde editie NL. Handleiding en verantwoording. In: Wechsler Intelligence Scale for children in Dutch. Manual and justification. 3. ed. London: Harcourt, 2005.

KAUFMAN, A. S.; KAUFMAN, N. L. KBIT: Kaufman intelligence test. Madrid: Pearson, 2011.

KINTSCH, W. The construction-integration model of text comprehension and its implications for instruction. In: RUDDELL, R. B.; UNRAU, N. J. (Eds.). Theoretical models and processes of reading. 5. ed. Newark, NJ: International Reading Association, 2004, p. 12701328.

KOSKI, J.; OLSON, I. R.; NEWCOMBE, N. S., Tracking the eyes to see what children remember. Memory, v. 21, v. 3, p. 396-407, 2013. DOI: 10.1080/09658211.2012.735241

LAGUN, D.; HSIEH, C.H.; WEBSTER, D.; NAVALPAKKAM, V. Towards better measurement of attention and satisfaction in mobile search. In: International ACM SIGIR Conference on Research \& Development in Information Retrieval, 37., 2014, Queensland, Proceedings [...]. Queensland: ACM Press, 2014, p. 113-122. DOI: $10.1145 / 2600428.2609631$

LEEW, L.; SEGERS, E.; VERHOEVEN, L. Role of text and student characteristics in realtime reading processes across the primary grades. Journal of Research in Reading, v. 39, n. 4, p. 389-408, 2015. DOI: 10.1111/1467-9817.12054

LVERSEDGE, S.P.; RAYNER, K.; WHITE, S.J.; FINDLAY, J.M.; Mc SORLEY, E. Binocular coordination of the eyes during reading. Current Biology, v. 16, p. 1726-1729, 2006. DOI: 10.1016/j.cub.2006.07.051

LITZINGER, T. A.; LEE, S. H.; WISE, J. C.; Felder, R. M. A Psychometric Study of the Index of learning styles. Journal of Engineering Education, v. 6, n. 4, p. 309-319, 2007. DOI: $10.1002 / \mathrm{j} .2168-9830$

MASON, L.; PLUCINO, P.; TORNATORA, M. C. Eye-movement modeling of text and picture integration during reading: effects on processing and learning. Contemporary Educational Psychology, v. 14, p. 172-187, 2013. DOI: 10.1007/11145-015-9552-5 
MOLINA, A. I.; REDONDO, M. A.; LACAVE, C.; ORTEGA, M. Assessing the effectiveness of new devices for accessing learning materials: An empirical analysis based on eye tracking and learner subjective perception. Computers in Human Behavior, v. 31, p. 475-490, 2014. DOI: $10.1016 /$ j.chb.2013.04.022

MORRISON, R. E.; Rayner, K., Saccade size in reading depends upon character spaces and not visual angle. Perception \& Psychophysics, v. 30, p. 395-396, 1981. DOI:

10.3758/BF03206156

POLLATSEK A.; REICHLE, E.D.; RAYNER, K., Tests of the E-Z Reader model: Exploring the interface between cognition and eye movements. Cognitive Psychology, v. 1, p. 52-56, 2006. DOI: 10.1016/j.cogpsych.2005.06.001

POOLE, A.; BALL, L. Eye tracking in human-computer interaction and usability research: current status and future. In: Encyclopedia of Human Computer Interaction, 2005.

RAYNER, K. Eye movements in reading and information processing: 20 years of research. Psychological Bulletin, v. 124, p. 372-422, 1998. DOI: 0033-2909/98

RAYNER, K. Eye Movements in attention in reading scene perception and visual search. Quaterly Journal Exp. Psychol, v. 62, n. 8, p. 1457-506, 2009. DOI: 10.1080/17470210902816461

RAVEN, J. Manual for Raven's progressive matrices and vocabulary scales. Research supplement $\mathrm{n}$. 1: The 1979 British standardisation of the standard progressive matrices and Mill Hill vocabulary scales, together with comparative data from earlier studies in the UK, US, Canada, Germany and Ireland. San Antonio, Texas: Harcourt Assessment, 1981.

REICHLE, E.; POLLATSECK, A.; FISCHER, D.; RAYNER, K. Toward a model of eye movement control in reading. Psychological Review, v. 105, p. 125-157, 1998. PMID: 9450374

REICHLE, E.D.; RAYNER, K.; POLLATSECK, A. The E-Z Reader model of eyemovement control in reading: Comparisons to other models. Behavioural and Brain Sciences, v. 26, p. 445-476, 2003. PMID: 15067951

REICHLE, E.; DRIEGHE, D. Using E-Z Reader to examine the consequences of fixationlocation measurement error. Journal of Experimental Psychology: Learning, Memory, and Cognition, v. 41, p. 262-270, 2015. DOI: 10.1037/a0037090

TIU, R. D. Jr.; THOMPSON, L. A.; LEWIS, B. A. The role of IQ in a component model of reading. Journal of Learning Disabilities, v. 36, n. 5, p. 424-436, 2003. DOI: $10.1177 / 00222194030360050401$

TOBII. Eye tracking. Disponível em: http:/ /www.tobii.com/. Acesso em: 25 jan. 2019.

WILLIAMS, R.S.; MORRIS, R. K. Eye movements, word familiarity, and vocabulary acquisition. European Journal of Cognitive Psychology, v. 16, p. 312-339, 2004.

VAN DEN BROEK, P.; RAPP, D. N.; KENDEOU, P. Integrating memory-based and 
constructionist approaches in accounts of reading comprehension. Discourse Processes, v. 39, p. 299-316, 2005. DOI: 10.1080/0163853X.2005.9651685

VAN, T.; SCHEITER, K. Eye tracking as a tool to study and enhance multimedia learning Learning and Instruction, v. 20, n. 2, p. 95-99, 2010. DOI:

10.1016/j.learninstruc.2009.02.009

VERHOEVEN, L.; VERMEER, A. Leeswoordenschat. Vocabulary for reading. Arnhem, the Netherlands: Cito, 1999.

VERHOEVEN, L.; KEUNING, J.; HORSELS, L.; VAN BOXTEL, L. Testinstrumentarium taalontwikkelingsstoornissenvoor kinderen van 4 tot 10 jaar. Arnhem, the Netherlands:

Cito, 2013.

\section{Como referenciar este artigo}

LEVRINI, Gabriel. Habilidades de leitura e competências de compreensão de texto de estudantes bolsistas oriundos de comunidades da Amazônia: estudo de caso com a utilização de Eye Tracker. Revista Ibero-Americana de Estudos em Educação, Araraquara, Araraquara, v. 15, n. 3, p. 1094-1114, jul./set. 2020. E-ISSN: 1982-5587. DOI: https://doi.org/10.21723/riaee.v15i3.12665

Submetido em: 11/06/2019

Revisões requeridas em: 20/08/2019

Aprovado em: 02/02/2020

Publicado em: 20/02/2020 